\title{
Coaxial Needle Insertion Assistant for Epidural Puncture
}

\author{
Yoshihiko Koseki, Danilo De Lorenzo, \\ Kiyoyuki Chinzei Member, IEEE, and Allison M. Okamura Fellow, IEEE
}

\begin{abstract}
Detection of internal puncture during manual needle insertion is challenging due to the large friction force between a needle shaft and surrounding tissue, which masks small changes in force at the needle tip. A novel robotic coaxial needle insertion assistant was developed to enhance operator perception during epidural puncture. The coaxial needle separates the cutting force at the needle tip from shear friction on the needle shaft. When the assistant is inactive, the human operator controls the motion of the coaxial needle. When the assistant is active under position control, a motor controls the motion of the outer needle, while the operator controls only the motion of the inner needle. When the assistant is active under force control, the operator controls the motion of the entire needle, but receives force feedback that is a scaled version of the force applied by the needle tip to the tissue. The effectiveness of the assistant in enabling puncture detection was tested in an experiment in which users were asked to puncture artificial tissues with the assistant inactive and active. Results show that the ratio of successful to unsuccessful puncture detection was higher with the assistant than without. In addition, users were more confident that they could perceive the moment of puncture. However, when the users successfully perceived puncture, overshoot with the assistant was larger than without.
\end{abstract}

\section{INTRODUCTION}

E PIDURAL anesthesia is a procedure to inject anesthetic drug into epidural space. The drug infiltrates spinal cord through dura matter, and blocks transmission of pain stimulus. An indwelling catheter can be placed into epidural space for a continuous infusion. Because of these features, epidural anesthesia is popular in medical procedures, especially in obstetrics.

For proper placement of the anesthetic drug, an epidural needle has to advance far enough to penetrate ligamentum flavum, but stop before the dura matter. Since the ligamentum flavum and dura matter are close to each other, a high level of

This work was supported by National Institutes of Health under Grant R01 EB006435, Japan Society for the Promotion of Science under Excellent Young Researcher Overseas Visit Program, Scuola Interpolitecnica di Dottorato, Johns Hopkins University, Advanced Industrial Science and Technology, and Politecnico di Milano.

Y. Koseki is with Research Institute of Human Life Technology, Advanced Industrial Science and Technology (AIST), 1-2-1 Namiki, Tsukuba, Ibaraki 305-0031, Japan (e-mail: koseki\{atmark\}ni.aist.go.jp).

D. De Lorenzo is with Politecnico di Milano, Bioengineering Department, Neuroengineering and Medical Robotics Laboratory, Milan, Italy.

K. Chinzei is with Research Institute of Human Life Technology, Advanced Industrial Science and Technology (AIST), Tsukuba, Japan.

A. M. Okamura performed this work in the Department of Mechanical Engineering, and the Laboratory for Computational Sensing and Robotics, Johns Hopkins University. She is now in the Mechanical Engineering Department, Stanford University. technical proficiency is required to place the needle tip in the epidural space. The loss of resistance (LOR) technique, in which the clinician stops when he feels a significant decrease in air/saline pressure in a syringe attached to the needle while advancing the needle, is the gold standard for identifying the epidural space. However in $25.7 \%$ of lumbar epidural steroid administration cases, LOR happens before the needle tip reaches the epidural space [1]. Due to this, the incidence of inadvertent dural puncture was reported as $0.04-6 \%$ [2]. If dural puncture occurs, the patient is likely to experience a post-dural puncture headache.

Many researchers have studied methods to improve the accuracy of epidural needle placement. Fluoroscopy [1], ultrasonography [3], and epiduroscopy [4] provided real-time monitoring of needle insertion. Tactile sensors and pressure sensors have been used to quantify the mechanism of LOR during lumbar puncture [5]. Spectroscopy has been used to enable the identification of tissue type [6]. Epidural injection simulators with haptic feedback have been developed to train clinicians, e.g. [7]-[9].

The sharp drop in cutting force that occurs at the moment of penetration of the ligamentum flavum is obfuscated by shear friction between the needle and surrounding tissue. This significantly complicates manual discrimination of the penetration. In a previous study, we used an instrumented coaxial needle to measure cutting force separately from shear friction force [10]. This instrumented coaxial needle operates as follows. The inner needle of the coaxial needle forms the front end of the needle, which cuts the tissue. The outer needle covers the side of the inner needle, preventing the shear friction between the tissue and outer needle from acting on the inner needle. A force sensor attached on the back end of the inner needle measures the drop in cutting force when the needle penetrates the tissue. The exact force profile depends on insertion speed, tissue properties, friction, and potentially other unknown factors. While previous work has focused on identification of penetration, we consider here how to display the cutting force to an operator during needle insertion in order to improve operator perception of penetration.

In this paper, we present a robotic coaxial needle insertion assistant that actively controls the needle such that the operator resists a force equal or proportional to the cutting force. It is expected that the operator can intuitively perceive the cutting force by inserting the needle as is done in conventional procedures. The contributions of this work are the design of the needle insertion assistant robot, and two control methods for the device. With the first method, the 


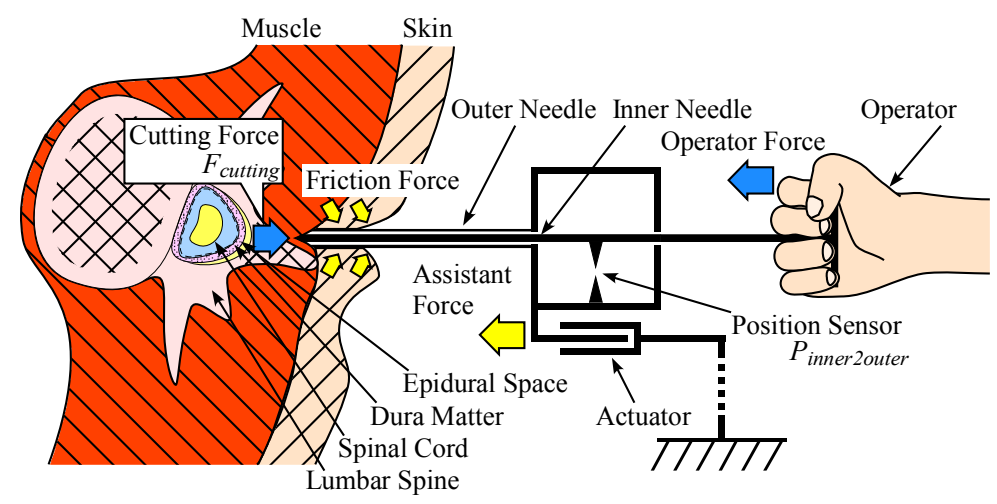

(A) Position-Controlled Assistant

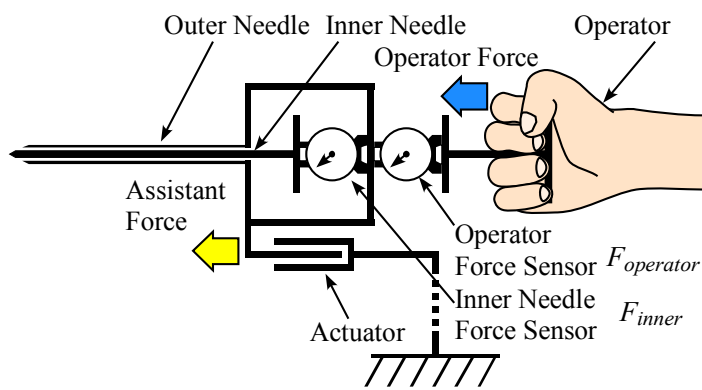

(B) Force-Controlled Assistant

Fig. 1. Overview of the coaxial needle insertion assistant. (A) In the position-controlled case, the user controls the motion of the inner needle, and the motion of the outer needle is controlled by a motor. (B) In the force-controlled case, force sensors are used to sense the force applied by the operator and the force applied by the inner needle to the tissue (at the tip). An actuator controls the motion of the outer and inner needle together such that the operator receives force feedback that is a scaled version of the force applied to the tissue.

cutting force was separated using position control. With the second method, the cutting force was amplified using force control. The effectiveness of these methods was tested in an experiment in which users inserted needles into artificial tissue, and attempted to stop when penetration (puncture) was detected.

\section{MAterials AND MethodS}

\section{A. Coaxial Needle Insertion Assistant Control}

Fig. 1(A) shows a coaxial needle inserted into the lumbar region of the spine with the position-controlled coaxial needle insertion assistant. Fig. 1(B) shows the force-controlled coaxial needle insertion assistant.

The front end of the inner needle cuts the tissue. As the needle is inserted into the tissue, shear friction occurs between the needle and the surrounding tissue. The outer needle covers the side of inner needle and prevents the shear friction from acting on the inner needle, while still allowing the inner needle to perform the cutting at the tip. This configuration allows the operator or a force sensor to detect the cutting force by pushing back end of the inner needle.

In case of the position-controlled assistant (Fig. 1(A)), the inner needle and outer needle are pushed by the operator and an actuator, respectively. A displacement sensor is placed between the inner needle and outer needle, and the actuator is position-controlled with the goal maintaining the relative position between the inner needle and outer needle as a constant. (The position error between the inner and outer needle, $P_{e}$, should ideally be zero.) This allows the outer needle to follow the movement of inner needle, and maintains the condition that the inner needle is covered by the outer needle. The operator inserts only the inner needle, which is mechanically isolated from the outer needle, so that the position-controlled assistant presents only cutting force to the operator. A PID (proportional-integral-derivative) algorithm was implemented to decide actuator output $\left(F_{a c t}\right)$ :

$$
F_{a c t}=k_{p} P_{e}+k_{i} \int P_{e}+k_{d} \dot{P}_{e}
$$

In the case of the force-controlled assistant (Fig. 1(B)), the inner and outer needles are jointed at the back end and inserted by the operator and actuator collectively. Force sensors are placed at the operator handle, and between the inner and outer needle. The actuator is force-controlled in an attempt to make the operator force $\left(F_{\text {operator }}\right)$ and cutting force measured by the inner needle $\left(F_{\text {inner }}\right)$ proportional $\left(F_{\text {operator }}=\right.$ $\left.\alpha F_{\text {inner }}\right)$. The force-controlled assistant can amplify the cutting force and present it to the operator. An implicit force set-point regulator-based force scaling algorithm [11] was implemented to determine the coaxial needle velocity $\left(V_{\text {needle }}\right)$. The velocity is controlled with a PID controller on position. The force gain $\alpha$ was set to 1.5 , the maximum value that could maintained stably with a wide variety of human operators.

In order to be sure that the human intention to move the needle forward or backward is not in conflict with the control algorithm, the following constraints were applied to the control algorithm:

$$
\begin{aligned}
& \text { if } F_{\text {operator }}>0 \text { and } V_{\text {needles }}>0 \\
& V_{\text {needle }}=k_{f}\left(F_{\text {operator }}-\alpha F_{\text {inner }}\right)
\end{aligned}
$$

Otherwise,

$$
V_{\text {needle }}=k_{f} F_{\text {operator }}
$$

Thus, the operator force is always in agreement with the actuator motion. During backward motion, the cutting force is not taken into account because it does not provide any relevant clinical information.

\section{B. Coaxial Needle Insertion Assistant Electromechanical Design}

Fig. 2 shows the detailed design and implementation of coaxial needle insertion assistant. The two parts of the coaxial needle were mounted on separate inner needle and outer needle sliders, respectively, and moved along a linear guide (2RSR9ZMUU +275LM, THK). The inner needle slider was equipped with a handle for the operator to push it. The outer 


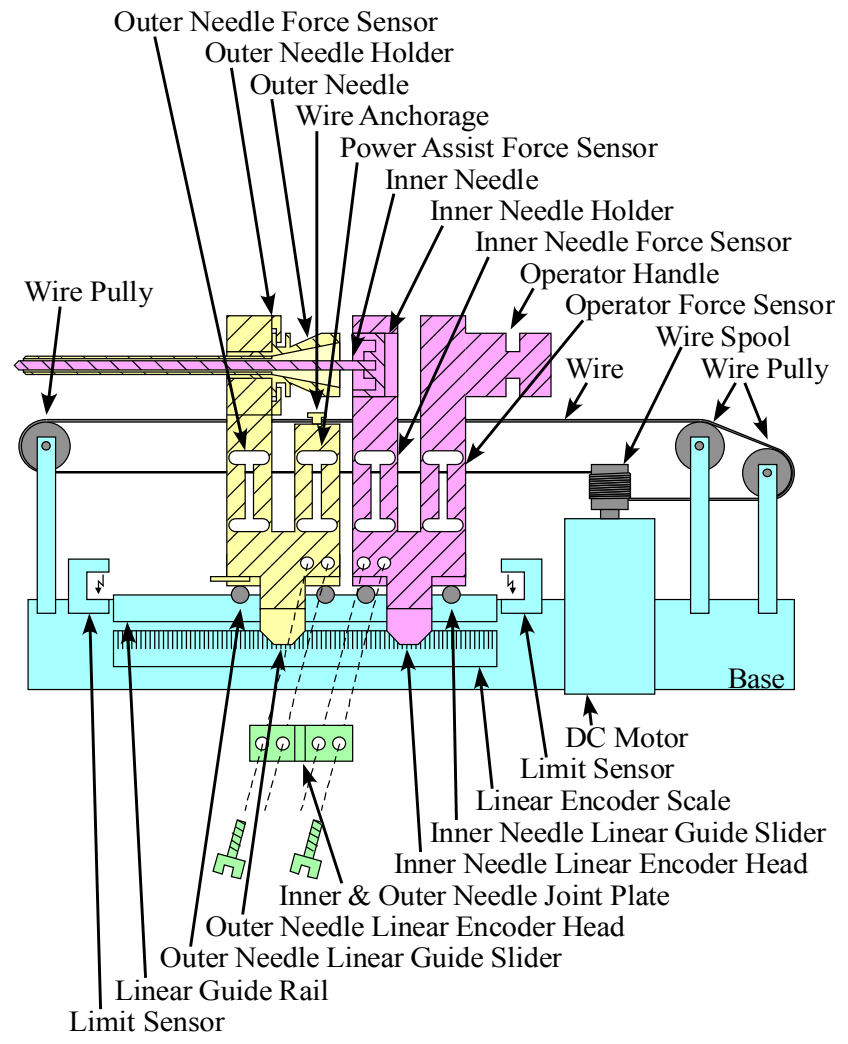

Fig. 2. Design and implementation of the coaxial needle insertion assistant. A wire driven by a DC motor is used to actuate the outer needle. For the force control condition, a plate is added to physically join the inner and outer needles. More force sensors are provided on the device than are required for control; the extra force sensors can be used to acquire system performance data during puncture experiments.

needle slider was driven via wire by a geared DC motor (41.022.022-00.00-202, Maxon). The wire was detachable from the slider. A joint plate could join the inner needle slider and outer needle slider, to be used during force-assistant and non-assistant (inactive) mode. The system has one degree of freedom of linear motion along the needle axis. The maximum specifications are $10 \mathrm{~mm} / \mathrm{sec}$ velocity, $10 \mathrm{~N}$ maximum force output, and $130 \mathrm{~mm}$ stroke.

Four force sensors were implemented for the inner needle holder, outer needle holder, operator handle, and wire anchorage. The one for inner needle was designed to measure $10 \mathrm{~N}$ maximum, and others were designed to measure $40 \mathrm{~N}$ maximum. The force sensor was a flexure parallelogram mechanism, and strain on the mechanism caused by load was measured by strain gauges. The strain gauges electrically formed a Wheatstone bridge and their outputs were amplified by instrumentation amplifier (INA118 and INA128, Texas Instruments). All force sensors were calibrated with weights (accuracy $20 \mu \mathrm{N}$ ). Force sensors and position sensors for inner needle, outer needle, operator, and actuator are used to investigate state of puncture; only the inner needle and operator force sensors are used in the device control. The displacements of the sliders were measured by optical linear encoders (LM-25CPMM-3S, Encoder Technology, 0.01mm

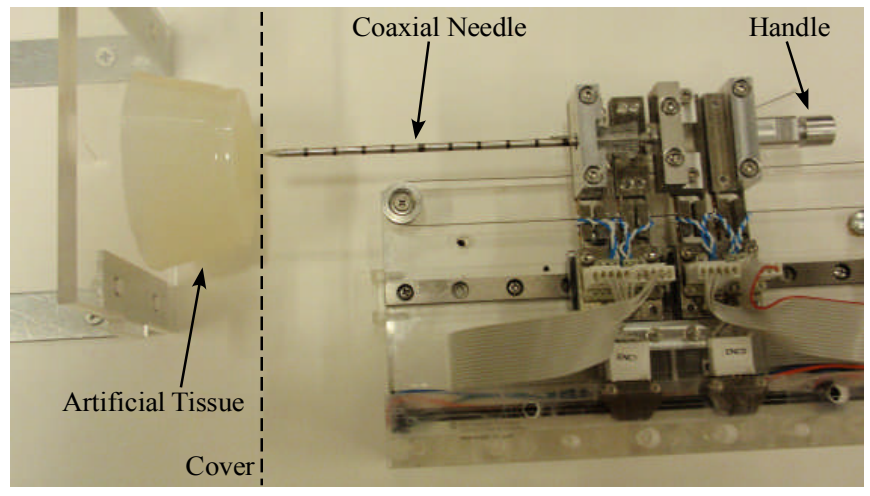

Fig. 3. Experiment setup. A dashed line shows the normal location of the artificial tissue cover, which is removed in this image.

resolution), sharing a single linear scale. The force signals, the position signals, and the control algorithm were acquired and refreshed at $1 \mathrm{msec}$ intervals by means of a real-time operating system (RT-Linux/GPL 3.2 rc1 with Linux kernel 2.4.29).

When the device is operating in non-assistant (inactive) mode, the wire is detached and the sliders are joined. Thus, the needles are mechanically isolated from the actuator and inserted only by the operator. When the device is operating as a position-controlled assistant, the wire is attached to the outer needle holder and the sliders are separated. Thus, the inner needle is inserted by the operator and the outer needle is inserted by actuator. When the device is operating in force-controlled assistant mode, the wire is attached to the outer needle holder and sliders are joined. In this case, the needles are inserted together by both the operator and actuator.

\section{User Experiment}

The goal of the experiment was to compare user performance during a needle insertion task under the three control modes (position control, force control, and non-assistant). Users were asked to insert a needle into artificial tissue, and to stop inserting as soon as they perceived the needle tip exiting the tissue (penetration/puncture). The overshoot of the needle past the exit point of the tissue was measured using a scale $(0.5 \mathrm{~mm}$ resolution). Users were also asked to rate their confidence in perceiving penetration after each trial.

Fig. 3 shows the experimental setup. Artificial tissues were made of silicone rubber with two different hardness values (Ecoflex H10 and H30, Smooth-on, where the H-number represents the rubber hardness on the Shore 00 scale). The artificial tissues were cured in two different thicknesses: $30 \mathrm{~mm}$ and $40 \mathrm{~mm}$. The artificial tissues were supported behind by an acrylic plate with a $\varnothing 25 \mathrm{~mm}$ hole to allow the needle to pass through. Two steel coaxial needles with different diameters designed for biopsy were used (C2016B and C1616B, Bard: 19G and 15G, respectively).

Nine right-handed, neurologically healthy, 
non-medical-professional users participated in this study. This study was approved by the Johns Hopkins University Homewood Institutional Review Board. The users were seated in front of the experimental setup. Holding the handle with the right hand, they punctured the artificial tissues with coaxial needles. The speed of insertion was voluntarily decided by each user. The tissue was covered except for the entry point of the needles, so that the users could not observe the back of the tissue where the needle exited. Each user performed one needle insertion under each combination of three assistant types (position control, force control, and non-assistant), two needle sizes (19G and $15 \mathrm{G}$ ), and four tissue types (combinations of two hardnesses and two thicknesses). Thus, the total number of insertions performed by each user was 24 . The experiment was designed to evaluate the immediate impact of different assistant types, but not to measure the learning aspect of needle insertion. To equalize user adaptability to needle insertion, the order of insertion was sequenced as randomly as possible, given the experimental setup. Four artificial tissues were punctured in pseudo-random order after a certain assistant type and needle type were selected. The order of assistant types was changed randomly between users and that of needles was changed randomly within assistant type. Before the actual session, the users practiced puncturing tissue approximately three times in each condition. The practice session was also sequenced pseudo-randomly.

For each insertion, the overshoot length (S) out of the tissue sample was measured using a scale. If the user stopped at the moment of puncture, the overshoot would be small. The users rated their confidence in perceiving the needle tip exiting the tissue on a scale of "No", "Low", and "High".

\section{RESULTS}

Fig. 4 shows the frequency distribution of needle overshoot under the different types of controllers: non-assistant,

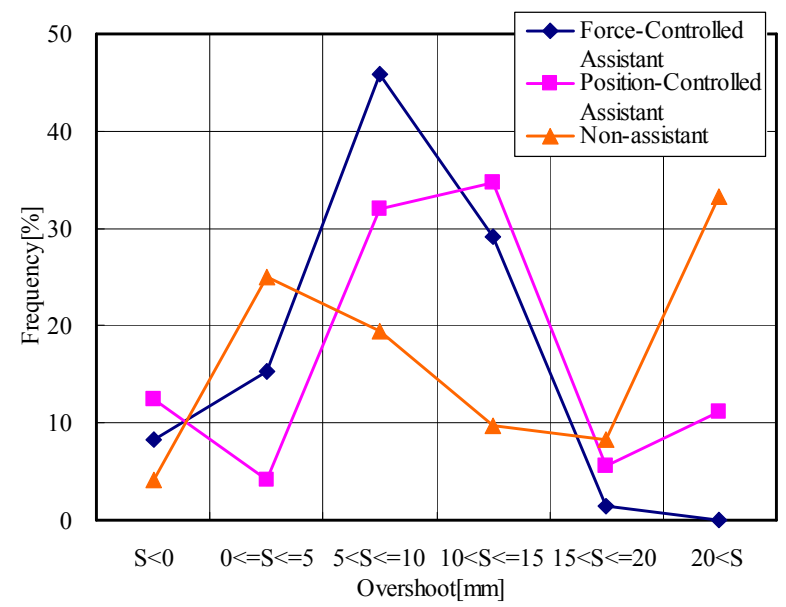

Fig. 4. Frequency of needle's overshoot, with position-controlled assistant, force-controlled assistant, and non-assistant. position-controlled assistant, and force-controlled assistant. The other conditions (thickness of tissue, hardness of tissue, and needle type) are summed for each controller type. Each trial was classified depending on the level of overshoot (S), with the width of a class defined as $5 \mathrm{~mm}$. Two special cases, failure to penetrate $(S<0)$ and failure to perceive any penetration $(20<\mathrm{S})$, were also defined as classes. With no assistance, users failed to perceive penetration with a higher frequency and this resulted in large overshoot. (Users eventually stopped after realizing they missed the penetration, or they reached the end of the workspace of the device.) With the position-controlled assistant and force-controlled assistant, the users failed much less frequently. Comparing the trials in which the users succeeded in perceiving penetration $(0<=S<=20)$, overshoot with the non-assistant was smaller than with the position- or force-controlled assistant, and overshoot with the force-controlled assistant was smaller than with the position-controlled assistant.

Fig. 5 shows the frequency distribution of needle overshoot with respect to the different types of assistance, taking into account the level of user confidence in perceiving the tissue penetration. Considering the different hardness of the samples (H10 and H30), both with position- and force-controlled assistance, the rate of successful insertion $(0<S<=10)$ perceived with high confidence from the user increases with the tissue hardness. In the case of unassisted insertion, if the penetration was perceived by the user, the overshoot is higher in the case of harder tissue.

Fig. 6 shows the confidence users had in performing a successful insertion. Here, successful insertion was defined as an insertion whose overshoot was less than $10 \mathrm{~mm}(0<\mathrm{S}<=10)$. This threshold is larger than the actual epidural space (approximately $5 \mathrm{~mm}$ ). Considering the discrepancies between our experimental setup and clinical epidural needle insertion (in particular, needle types, tissues, supporting condition of tissue, and user expertise), the threshold was defined independently of a clinically relevant value in order to best illustrate differences between the conditions. With non-assistant, $44 \%$ of insertions were successful, but only $34 \%$ of them were done with high confidence. On the other hand, with the position-controlled assistant, $36 \%$ of insertions

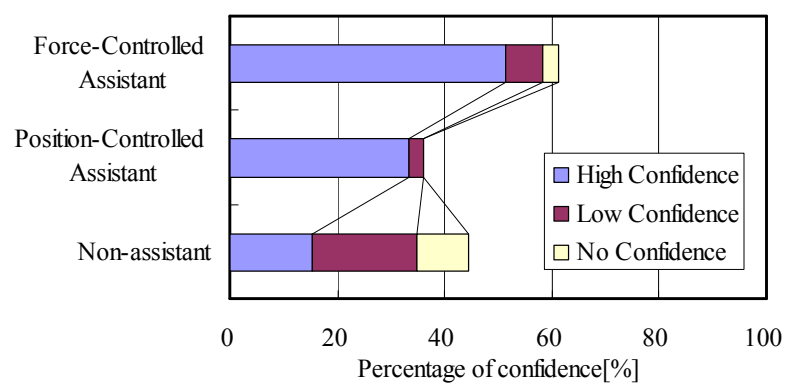

Fig. 6. User confidence in successful insertions. For each successful insertion, users were asked to rate their confidence that they felt the moment of penetration/puncture. 

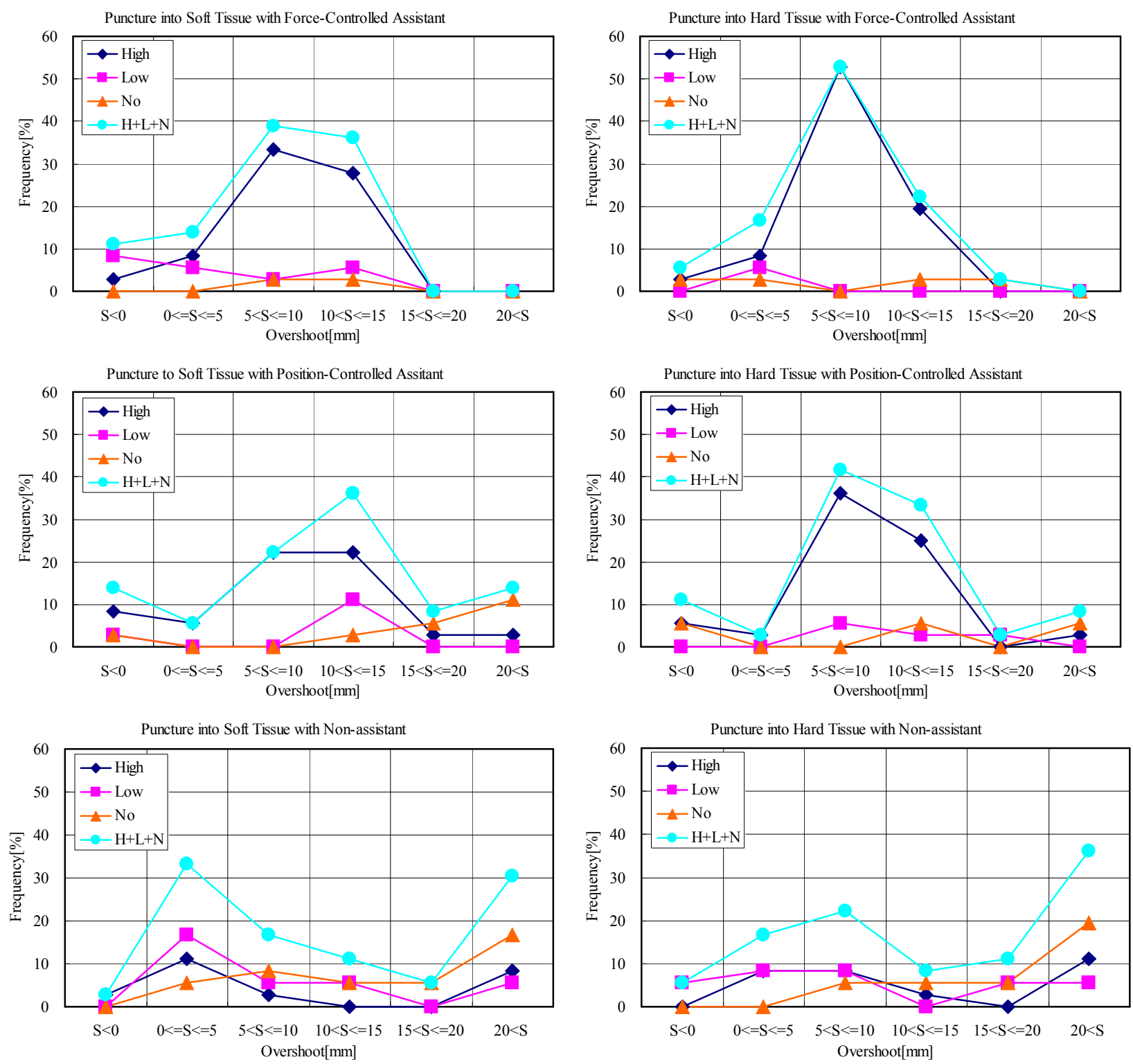

Fig. 5. Frequency of the needle overshoot, with force-controlled assistant, position-controlled assistant, and non-assistant for the two types of artificial tissue hardness: soft (H10) and hard (H30). The different traces within each plot indicate the level of user confidence in detecting puncture (High, Low, None, and all confidences combined).

were successful but $92 \%$ of them were done with high confidence. With the force-controlled assistant, $61 \%$ of insertions were successful, and $84 \%$ of them were done with high confidence.

\section{DISCUSSION AND CONCLUSION}

It was experimentally shown that the coaxial needle assistant facilitated the perception of penetration during needle insertion. The force-controlled assistant was more effective than the position-controlled assistant at helping users notice the penetration event, although both performed better than no assistance. The force-controlled assistant both presents the cutting force 1.5 times larger than the position-controlled assistant and helps remove the perception of friction force between the inner and outer needle and between the sliders. Thus, users could detect the drop in cutting force at the moment of penetration more easily.

Harder artificial tissue samples generally resulted in less overshoot than softer samples. This can be explained considering the higher friction forces along the outer needle resulting from the harder samples, which prevent higher overshoot with respect to softer samples. This agrees with the relatively better performance of the unassisted insertion in soft tissue compared to unassisted insertion into hard tissue, where the higher friction forces between the outer needle and the tissue mask penetration forces and decrease user sensitivity to penetration. This intuitively follows from Weber's Law, which states that the ratio of the increment threshold to the 
background intensity is a constant. When the background intensity is larger due to friction, larger changes in cutting force are required for the user to perceive puncture.

When the users successfully perceived penetration, the overshoot in the position-controlled and force-controlled assistant conditions were larger than those in the non-assistant condition. This occurs because a user cannot react sufficiently quickly to the rapid drop in the cutting force and thus inserted the needle deeper before stopping. (When a user is applying a large force against a resistance, and the resistance is suddenly removed, the user has difficulty stopping the unexpected forward motion.) This is not surprising, given previous results [12] demonstrating that users can have more needle insertion overshoot with haptic feedback than without. This situation can be improved by changing the control parameters.

Friction between the inner and outer needles degraded the haptic feedback to the user in the position-controlled assistant condition, since the user felt not only the cutting force at the tip of the needle, but also friction force from the outer needle moving relative to the inner needle. The apparatus can be improved to ensure better alignment of the inner and outer needle. In addition, improving the motion control of the outer needle would decrease position errors between the two needles. Increasing the air gap between the needles is undesirable, as this would increase the overall size of the coaxial needle. Another source of friction in system is in the linear guides. This affected forces felt by the user in the position-controlled assistant and non-assistant cases, although the effect is smaller in the position control case, when the user only moves the inner needle with respect to ground. In the case of the force-controlled assistant, the amount of the friction felt by the user depends on the effectiveness of the force controller. Decreasing the friction is in general expected to improve the assistant.

In clinical practice, a lateral force will be applied when the physician tries to change needle's trajectory and/or when the tissue is deformed in lateral direction. This might bend the needle and increase the friction between the inner and outer needles. In this experiment, only forces along needle were considered and therefore the friction between needles was small (roughly $0.2 \mathrm{~N}$ ) in comparison with the cutting force $(1.5 \mathrm{~N})$. Further study is necessary to reveal and suppress the effect of the friction between needles.

Overall, the results indicate that a force-controlled assistant is better at facilitating perception of penetration than the position-controlled assistant. However, position control can be considered safer due to simpler stability conditions and lack of susceptibility to force sensor noise, so position control is likely to be more suitable for medical applications. Another practical consideration is that position control does not require an additional force sensor to measure needle-tissue contact forces and the hand user forces.

Both assistant types should be comprehensively tested in preclinical conditions, such as using ex vivo biological tissue and needles matching the epidural procedure, to better evaluate clinical relevance. In addition, design modifications of the system to enable delivery of therapy, requiring removal of the inner needle and insertion of anesthesia (and potentially a catheter), should also be considered.

\section{REFERENCES}

[1] W. S. Bartynski, S. Z. Grahovac, and W. E. Rothfus, "Incorrect Needle Position during Lumbar Epidural Steroid Administration: Inaccuracy of Loss of Air Pressure Resistance and Requirement of Fluoroscopy and Epidurography during Needle Insertion", American Journal of Neuroradiology, Vol. 26, pp. 502-505, 2005.

[2] C. W. Berger, E. T. Crosby, W. Grodecki, "North American survey of the management of dural puncture occurring during labour epidural analgesia", Canadian Journal of Anesthesia, Vol. 45, No. 2, pp. 110-114, 1998.

[3] D. Tran, A. A. Kamani, E. Al-Attas, V. A. Lessoway, S. Massey, R. N. Rohling, "Single-operator real-time ultrasound-guidance to aim and insert a lumbar epidural needle", Canadian Journal of Anesthesia, Vol. 57, No. 4, pp. 313-321, 2010.

[4] T. Igarashi, Y. Hirabayashi, R. Shimizu, K. Saitoh, H. Fukuda, H. Suzuki, "The Fiberscopic Findings of the Epidural Space in Pregnant Women", Anesthesiology, Vol. 92, Issue 6, pp. 1631-1636, 2000.

[5] D. Tran, KW Hor, A. A. Kamani, V. A. Lessoway, and R. N. Rohling, "Instrumentation of the Loss-of-Resistance Technique for Epidural Needle Insertion", IEEE Transactions on Biomedical Engineering, Vol. 56, No. 3, pp. 820-827, 2009.

[6] P. N. Brett, A. J. Harrison, and T. A. Thomas, "Schemes for the Identification of Tissue Types and Boundaries at the Tool Point for Surgical Needles", IEEE Transactions on Information Technology in Biomedicine, Vol. 4, No. 1, pp. 30-36, 2000.

[7] A. P. Daykin, R. J. Bacon, "An Epidural Injection Simulator", Anaesthesia, Vol. 34, pp. 235-236, 1990.

[8] P. N. Brett, T. J. Parker, A. J. Harrison, T. A. Thomas, A. Carr, "Simulation of resistance forces acting on surgical needles", Proceedings of the Institution of Mechanical Engineers, Vol. 211, No. 4, pp. 335-347, 1997.

[9] J. C. Magill, M. F. Byl, M. F. Hinds, W. Agassounon, S. D. Pratt, P. E. Hess, "A Novel Actuator for Simulation of Epidural Anesthesia and Other Needle Insertion Procedures", Simulation in Healthcare, Vol. 5 , No. 3, pp. 179-184, 2010.

[10] T. Washio, and K. Chinzei, "Needle Force Sensor, Robust and Sensitive Detection of the Instant of Needle Puncture", Proceedings of the International Conference on Medical Image Computing and Computer Assisted Intervention (MICCAI), Lecture Notes in Computer Science Vol. 3217, pp. 113-120, 2004

[11] J. Roy, D. L. Rothbaum, and L. L. Whitcomb, "Haptic Feedback Augmentation through Position Based Adaptive Force Scaling: Theory and Experiment", Proceedings of IEEE/RSJ International Conference on Intelligent Robots and Systems (IROS), pp. 2911-2919, 2002.

[12] O. Gerovich, P. Marayong, and A. M. Okamura, "The Effect of Visual and Haptic Feedback on Computer-Assisted Needle Insertion", Computer-Aided Surgery, Vol. 9, No. 6, pp. 243-249, 2004. 